\title{
Una excepción en la trayectoria formativa de las mujeres: Al-Ándalus en los siglos VIII-XII
}

\section{An exception in the academic training of women: Al- Andalus during the 8th to the 12th centuries \\ Uma exceção na formação das mulheres: Al-Andalus nos séculos VIII-XII}

Blanca Espina-Jerez ${ }^{1}$, Patricia Domínguez-Isabel², Sagrario GómezCantarino ${ }^{3}$, Paulo Joaquim Pina-Queirós ${ }^{4}$, Carmen Bouzas-

Mosquera $^{5}$

${ }^{1}$ Universidad de Castilla-La Mancha. Campus Toledo. Graduada en Psicología. MSc en Psicología Clínica y de la Salud. Grado en Enfermería. Estudio Asociado HISAG-EP. Correo electrónico: b.espinajerez@gmail.com ${ }^{2}$ Universidad de Castilla-La Mancha. Campus Toledo. Grado en Enfermería. Estudio Asociado HISAG-EP. Correo electrónico: patriventas98@gmail.com ${ }^{3} \mathrm{PhD}$ Universidad de Castilla-La Mancha, Toledo. Departamento: Enfermería, Fisioterapia y Terapia Ocupacional. Campus de Toledo. Correo electrónico: sagrario.gomez@uclm.es

${ }^{4}$ PhD Professor Coordinador. Escola Superior de Enfermagem de Coimbra, Portugal. Estudio Asociado HISAG-EP. Correo electrónico: pauloqueiros@esenfc.pt

${ }^{5} \mathrm{PhD}$, Universidad de Castilla-La Mancha, Toledo. Departamento: Enfermería,

Fisioterapia y Terapia Ocupacional. Estudio Asociado HISAG-EP. Correo electrónico: carmen.bouzas@uclm.es

Cómo citar este artículo en edición digital: Espina-Jérez, B., Domínguez-Isabel, P., Gómez-Cantarino, S., Pina-Queirós, P.J. \& Bouzas-Mosquera, C. (2019). Una excepción en la trayectoria formativa de las mujeres: Al-Ándalus en los siglos VIII-XII. Cultura de los Cuidados (Edición digital), 23 (54). Recuperado de $h$ ttp://dx.doi.org/10.14198/cuid.2019.54.17

Correspondencia: Sagrario Gómez-Cantarino. Universidad de Castilla - La Mancha, Toledo. Departamento: Enfermería, Fisioterapia y Terapia Ocupacional

Correo electrónico de contacto: sagrario.gomez@uclm.es Recibido: 20/01/2019; Aceptado: 16/05/2019

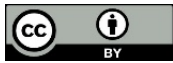

\section{ABSTRACT}

Introduction: Women in Al-Andalus, 8th to the 12th centuries, could get trained in a formal way in medicine and caring. This turning point between legal-religious regulation and healthcare was the result of different factors.

Main objective: To clarify the training course of women in Al-Andalus during the 8 th to the 12th centuries. Specific objectives: to know the educational beginnings of women in Al-Andalus; to establish the healthcare training paths of women in AlAndalus; to define the feminine figures dedicated to healthcare according to their academic education. 
Methods: Historical and descriptive review. It has conducted a bibliographic research in primary sources, original articles, manuals, libraries, historical archives and databases.

Results: The education of the andalusi women began with the reading and writing of the Koran. The training in healthcare was associated to male teachers known as tabib, and female teachers, tabiba and qabila. When their training was finished, women should acquire the iyaza and pass a theoreticalpractical exam. Andalusí women got trained in hispanic-arabic medicine.

Conclusions: The training of tabibas and qabilas in Al-Andalus was silent but revolutionary, giving its formal and regulated nature. They made contributions to the obstetrics. Part of the training tradition of Al-Andalus lasts nowadays.

Keywords: History of nursing, history of medicine, women physicians, teaching, cultural diversity.

\section{RESUMO}

Introdução: Considerando diferentes de lugares e períodos históricos, a mulher residente em Al-Andalus, durante os séculos VIII-XII, podia ser formada, de forma regulamentada, em medicina e em cuidado. Tal inflexão entre as normas jurídico-religiosas e a prática sanitária foi devida a diferentes fatores.

Objetivo geral: Esclarecer a trajetória formativa das mulheres de Al-Andalus durante os séculos VIII-XII. Objetivos específicos: conhecer os inícios formativos das mulheres em Al-Andalus; estabelecer os percursos de formação em saúde para as mulheres andaluzes; determinar as figuras femininas dedicadas aos cuidados de saúde em Al-Andalus de acordo com a sua formação.

Metodologia: Revisão histórico-descritiva. Pesquisa bibliográfica em fontes primárias, artigos originais, manuais e outros, bibliotecas, arquivos históricos e bases de dados.

Resultados: A educação da mulher andaluza começou com o aprendizagem da leitura e escrita do Corão. A formação em saúde esteve vinculada a professores do sexo masculino, tabib e do sexo feminino, tabiba e qabila. Quando terminaram a sua formação, tiveram que adquirir o iyaza e passar num exame teórico-prático. A sua formação foi em medicina hispano-árabe.

Conclusões: A formação de tabibas e qabilas andaluses foi silenciosa, mas revolucionária, dado o seu caráter regulado e formal, e contribuiu para a obstetrícia da época. Parte da tradição formativa em Al-Andalus dura até hoje.

Palavras chave: História da enfermagem, história da medicina, mulheres médicas, educação, diversidade cultural.

\section{RESUMEN}

Introducción: Diferenciando lugares y períodos históricos, la mujer residente en AlÁndalus durante los siglos VIII-XII pudo formarse de manera reglada en la medicina y el cuidado. Tal inflexión entre normativa jurídico-religiosa y práctica sanitaria se debió a diferentes factores.

Objetivo general: Esclarecer el recorrido formativo de las mujeres de Al-Ándalus durante los siglos VIII-XII. Objetivos específicos: conocer los inicios formativos de las mujeres en Al-Ándalus; establecer las vías de formación en salud de la mujer andalusí; determinar las figuras femeninas resultantes dedicadas a la atención sanitaria en Al-Ándalus según su formación.

Metodología: Revisión históricodescriptiva. Búsqueda bibliográfica en fuentes primarias, artículos originales, manuales y otros, bibliotecas, archivos históricos y bases de datos. 
Resultados: La educación de la mujer andalusí comenzó con el aprendizaje de la lecto-escritura del Corán. La formación en salud estuvo vinculada a docentes masculinos, tabib, y femeninas, tabiba y qabila. Cuando terminaban su formación, debían adquirir la iyaza y superar un examen teórico-práctico. Se formaron en medicina hispano-árabe.

Conclusiones: La formación de tabibas y qabilas andalusíes fue silenciosa pero revolucionaria, dado su carácter reglado y formal, e hizo aportaciones a la obstetricia de la época. Parte de la tradición formativa en Al-Ándalus perdura hasta la actualidad.

Palabras clave: Historia de la enfermería, historia de la medicina, mujeres médicas, enseñanza, diversidad cultural.

\section{INTRODUCCIÓN}

A pesar de que históricamente y de manera casi universal los cuidados han sido relegados a la mujer (Martín, Pérez \& Sabado, 2012), su aplicación no siempre ha estado fundamentada en una trayectoria formativa, y mucho menos han adquirido el reconocimiento sanitario, social y cultural merecido. En este sentido, la mujer residente en Al-Ándalus durante los siglos VIII-XII constituye una excepción (Cabanillas, 2012).

Desde la invasión árabe de la Península Ibérica en el año 711 d.C., en Al-Ándalus hubo una gran variedad étnica y cultural (Mesned, 2007). El territorio estuvo habitado por árabes, beréberes (procedentes del norte de África), Al-Mawälis (musulmanes no árabes) ${ }^{1}$, población autóctona hispanovisigoda, muladíes (cristianos convertidos al islam), judíos, esclavos (procedentes del norte de Europa y España) y normandos (llegaron con las invasiones de las costas andalusíes) (Guichard, 1998; Halilović, 2017; Mesned, 2007). Conforme la población musulmana fue adentrándose en la península, se sucedieron los procesos de arabización e islamización, es decir, la implantación del árabe como lengua y el islam como religión, para todos los pueblos presentes en ese momento (Abellán, 2006).

\section{FIGURA 1: Mapa de la expansión musulmana en la Península Ibérica, siglo VIII}

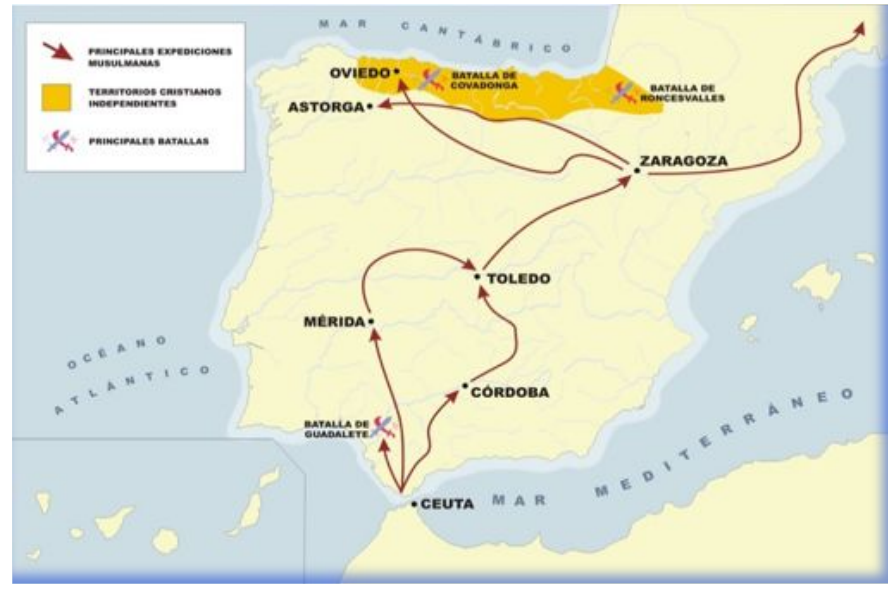

FUENTE: Banco de imágenes y sonidos. Ministerio de Educación y Formación Profesional. Gobierno de España. Disponible en: http://recursostic.educacion.es/bancoimagenes/web/ 
Resulta conveniente a su vez aclarar que el territorio andalusí llegó a ocupar casi toda la Península Ibérica, con la pequeña excepción de algunos reinos del norte (Figura 1). Tal puntualización se vuelve relevante para evitar confusiones con la actual Andalucía, Comunidad Autónoma española que comparte similitudes en la denominación, debido a que guardó durante más tiempo la diversidad cultural gestada en este momento histórico.

La elección del período histórico de este estudio estuvo determinada por la ocupación islámica de la región y por la propia delimitación práctica necesaria a la hora de estudiar la historia. La invasión árabe se materializó muy rápidamente en la península, puesto que en $716 \mathrm{~d}$. C. los árabes ya habían alcanzado el norte de España y empezaban a dirigir sus fuerzas hacia los Pirineos. Aunque los inicios de la Reconquista española se iniciaron años después, concretamente con la batalla de Covadonga en el $722 \mathrm{~d}$. C., los cristianos no consiguieron alcanzar más de la mitad peninsular hasta llegado el siglo XII, con lo que la ocupación árabe del territorio fue prolongada (Domínguez, 2013; Valdeón, 2006) (Figura 2).

\section{FIGURA 2: Mapa de la expansión de los reinos cristianos durante la Reconquista, siglo XII}

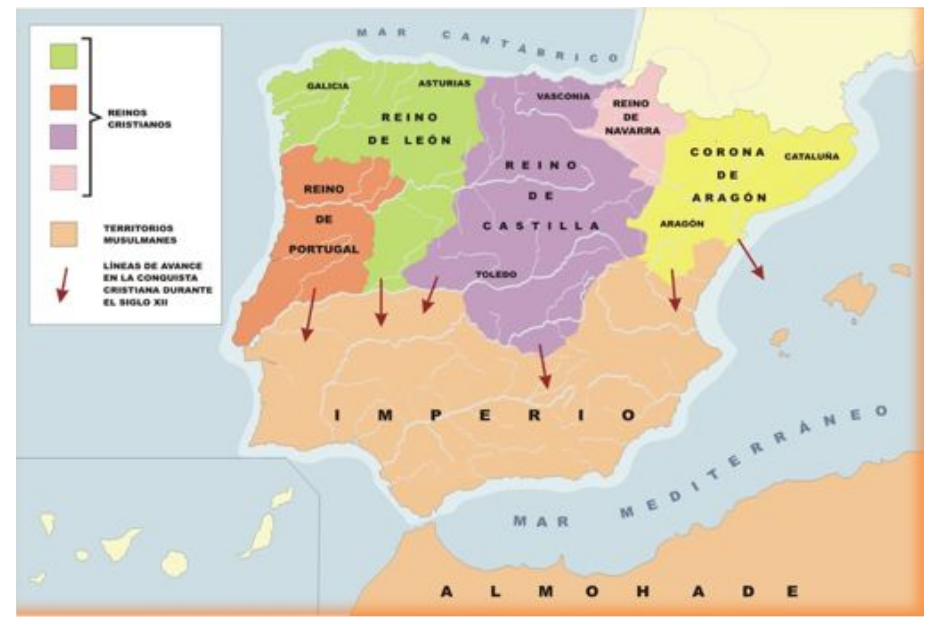

FUENTE: Banco de imágenes y sonidos. Ministerio de Educación y Formación Profesional. Gobierno de España. Disponible en: http://recursostic.educacion.es/bancoimagenes/web/

La religión y el proceso de islamización jugaron un papel muy importante en la configuración de la colectividad andalusí, al decidir de qué manera se regulaban los matrimonios entre las diferentes culturas (López, 1992). Al comienzo de la invasión árabe (siglo VIII), las restricciones se impusieron sobre todo en la mujer musulmana que venía de oriente, pues a ésta el derecho malikí le prohibía contraer matrimonio con un hombre cristiano o judío. Sin embargo, el hombre musulmán, es decir, el invasor, sí podía casarse con una mujer cristiana o judía, siempre bajo el compromiso de convertirse al islam en los meses posteriores, pues de lo contrario el matrimonio quedaría anulado. La descendencia de los matrimonios 
interculturales fue dando lugar a la población andalusí, educada desde el nacimiento en el islam (de Castro, 2015). Por este motivo, a partir de ahora el manuscrito utilizará de forma intercambiable los términos "mujer" y "mujer andalusí" para describir a las mujeres que residieron en el territorio de Al-Ándalus durante los siglos VIII-XII, y que fueron el resultado de la integración de diferentes culturas $y$ sociedades. Si bien sobre los documentos jurídico-religiosos la sociedad andalusí seguía un modelo patriarcal, se dio paso a una flexibilidad mayor en los roles $y$ patrones relacionales de la época, específicamente, en lo concerniente a la mujer. A partir del siglo $X$ se empezó a valorar la cultura, inteligencia $y$ conocimiento de la mujer andalusí, y no tanto su belleza y juventud (Cabanillas, 2012).

La mujer andalusí tomó un importante papel en distintas áreas culturales y sociales, en muchos casos independientemente de su condición social (Ávila, 1989). No obstante, la literatura en general tiende a vislumbrar una imagen encorsetada de la mujer de la época, en la que más bien tienen cabida los estereotipos de las poetisas, cantoras $y$ dedicadas a otros entretenimientos. Pero nada más lejos de la realidad, también existieron mujeres dedicadas las ciencias sociales y sanitarias, como la literatura, la copia, la astronomía, el cálculo, la medicina y los cuidados reglados de la mujer y el niño (Aguilar, 1997). Si bien es cierto que en la literatura se trata el tema de la mujer andalusí dedicada a la salud (Aguirre, 1997; Ávila, 1989; Cabanillas, 2012; Marín, 2006; Olmo \& Vidal, 2012; Roldán et al., 2014), apenas existen trabajos que aborden los factores que propiciaron su formación en la medicina y el cuidado, ni el recorrido que podían tomar para su adquisición. Así, el objetivo general del presente estudio fue esclarecer el recorrido formativo de las mujeres de Al-Ándalus durante los siglos VIII-XII. Para ello, se marcaron los siguientes objetivos específicos: conocer los inicios formativos de las mujeres en AlÁndalus; establecer las vías de formación en salud de la mujer andalusí; determinar las figuras femeninas resultantes dedicadas atención sanitaria en Al-Ándalus según su formación.

\section{METODOLOGÍA Y FUENTES}

Se llevó a cabo una revisión históricodescriptiva con el fin de conocer la trayectoria formativa de las mujeres residentes en Al-Ándalus durante los siglos VIII-XII. Para ello, se analizaron los inicios educativos, las diferentes vías de formación en salud y las figuras resultantes dedicadas al cuidado y la atención de la saludenfermedad durante este período histórico.

En una búsqueda inicial, se llevó a cabo un acercamiento a la cuestión por medio de bases de datos electrónicas: PubMed, CINAHL, SciELO y Dialnet. Con la información obtenida, se realizó una segunda búsqueda en la biblioteca de la Universidad de Castilla-La Mancha, la biblioteca pública de Castilla-La Mancha y en los archivos histórico y provincial de Toledo. De este modo se obtuvieron fuentes primarias de la época, como textos médicos, escritos de poesía y recursos artísticos; artículos originales; manuales que abordan el tema de la situación socio-cultural en AlÁndalus y, más concretamente, de la mujer andalusí; y comunicaciones en congresos. La relación documental se resume en la tabla 1 .

Los criterios de inclusión aplicados a la revisión fueron: utilización de material procedente de fuentes primarias, artículos originales, manuales y comunicaciones en 


\section{Cultura de los Cuidados}

congresos ceñidas a la temática de la investigación; época de estudio comprendida entre los siglos VIII y XII; idiomas manejados por los investigadores: inglés, español y portugués; trabajos procedentes de disciplinas sanitarias y no sanitarias. Como criterios de exclusión se marcaron: textos ceñidos específicamente a la formación de la mujer andalusí en otras disciplinas diferentes a la medicina y el cuidado; material referido a la situación del hombre en Al-Ándalus; documentos de bajo rigor científico.

TABLA 1: Número de documentos revisados

\begin{tabular}{|l|c|}
\hline \multicolumn{1}{|c|}{ TiPO DE DOCUMENTO } & N.o REVISADOS \\
\hline Artículos de Revista & 64 \\
\hline Fuentes Primarias & 6 \\
\hline Manuales & 16 \\
\hline Comunicaciones en congreso & 6 \\
\hline Tesis doctorales & 2 \\
\hline
\end{tabular}

FUENTE: Creación propia de las autoras

Se procedió a la descripción, reconstrucción e interpretación de los hallazgos descubiertos, adoptando una perspectiva hermenéutica con el fin de recopilar e interpretar de la manera más objetiva posible la información encontrada. Seguidamente, la información se ordenó siguiendo un criterio cronológico que facilite la compresión de la evidencia histórica construida.

\section{RESULTADOS}

\section{Los inicios formativos de las mujeres en Al-Ándalus}

Uno de los factores claves que determinó la dedicación de las mujeres a las ciencias sociales y sanitarias fue su inmersión en la lectoescritura del Corán. Estos comienzos formativos no estuvieron mediados por razones de índole económica, condición social o género (Mesned, 2007), pero sí supusieron una inflexión entre la normativa legal del momento y la práctica social. Legalmente, se desaprobaba que las niñas asistieran a las escuelas coránicas, pero se tiene constancia de que existieron maestros que se opusieron ante tal regulación, y se dedicaron a enseñar a las niñas en la mezquita, desde el amanecer hasta el final del día sin ningún tipo de remuneración económica (Lachiri, 1993). Además, debido a la segregación por sexos de gran parte de los espacios públicos, se fomentó la creación de espacios de reunión similares a los de los varones, pero sólo para mujeres (LéviProvençal, 1953; Marín, 2010). Estas variaciones en las normas jurídico-religiosas posibilitaron que las mujeres andalusíes pudieran tener un lugar en la ciencia, la literatura, la poesía y la docencia (Aguilar, 1997).

Una vez que se daba el salto a la adquisición de una formación más específica, la situación se volvía diferente. 
En muchos casos, las mujeres avanzaron y adquirieron posiciones laborales impensables al estar vinculadas a una figura masculina, bien por haber nacido en el seno de una familia de nivel socioeconómico y/o académico-educativo alto, o por prestar sus servicios a hombres de poder. Dos ejemplos del primer caso lo constituyen Umm alHasan, quien se formó en la lectura coránica y la poesía, y más tarde adquirió grandes conocimientos en medicina a través de las enseñanzas de su padre; y Umm Amr bint Abī Marwān Ibn Zuhr, hija del reconocido médico andalusí Avenzoar (m. 557/1162) (Olmo \& Vidal, 2012). Un patrón que siguió la segunda modalidad fue Lubna (m. IV/X), experta en gramática, poetisa, copista $\mathrm{y}$ matemática. Trabajó como escritora para el califa Al-Hakam II y fue contratada por este como secretaria mayor de la biblioteca de Medina Azahara (Córdoba), lugar que reunió una gran cantidad de volúmenes de todas las ramas del saber (Ávila, 1989; Bernis, 1956; Cabanillas, 2012).

\section{Vías de formación, docentes para la enseñanza en salud y paradigma médico}

Para las mujeres de Al-Ándalus generalmente existían dos vías posibles de formación. Podían hacerlo con una persona dedicada a la medicina, habitualmente un varón, o con una mujer encargada de asistir a mujeres y menores. De tales opciones emergían las figuras de tabiba y qabila.

Las mujeres que tuvieron un padre $u$ otro varón de la familia dedicado a la medicina, se formaron como tabibas o médicas por la vía del linaje (Aguilar, 1997; Marín, 2006; Olmo \& Vidal, 2012). No obstante, también existía la posibilidad de que aprendieran los conocimientos teóricos a través de un tutor particular externo a la familia, caso bastante excepcional cuando el profesor era varón y la estudiante mujer, aunque posible en la práctica. Requería que algún pariente estuviera presente durante las lecciones o se posicionara una cortina entre ambos, además de que la familia contara con una situación de privilegio económico que le permitiera costear la docencia de su hija (Ávila, 1989).

Debido a la aparición de figuras pseudoprofesionalizantes en el campo de la salud y los cuidados, se crearon dos regulaciones. Por un lado, en caso de que la tabiba quisiera dedicarse a la docencia, que sólo podía impartirla a otras mujeres mientras se hiciera con velo, debía estar en posesión de la iyaza o licencia para transmitir lo aprendido, regulación que ya existía en otras profesiones (Ávila, 1989; Lachiri, 1993). Por otro lado, la tabiba debía someterse a un examen teórico-práctico llevado a cabo por el médico jefe del momento, quien firmaba o no la licencia de acreditación teórica y práctica (Aguirre, 1997).

Existieron otras vías formativas para las figuras médicas, como el maristán $\mathrm{u}$ hospital, y la escuela médica privada (Aguirre, 1997). Aunque apenas existen datos sobre las instituciones sanitarias en AlÁndalus (Fernández \& Mañá, 2016), se sabe que en el mundo islámico se empezaron a desarrollar instituciones médicas, como la madrasa (escuela) y el maristán. En estos lugares se llevaba a cabo la educación médica $y$ se practicaban cuidados universales (Edriss, Rosales, Nugent, Conrad \& Nugent, 2017; Micheli-Serra, 2002). A pesar de la supuesta idoneidad de estos centros para la adquisición de conocimientos sanitarios, en Al-Ándalus apenas se tiene constancia de varias almadrasas y maristanes, como el de Granada, también conocido como maristán nazarí, ambas instituciones de instauración posterior al periodo histórico del presente estudio (Fernández \& Mañá, 2016; Said- 
Farah, 2008).

El paradigma médico andalusí entre los siglos VIII y XV fue conocido como medicina hispano-árabe, resultado de la confluencia de tres corrientes médicas: la medicina monástica, practicada por médicos mozárabes; la medicina del profeta, ejercida por los musulmanes que llegaron a la Península; y la medicina greco-helenística, de inicio más tardío en Al-Ándalus y consecuencia de la asimilación de la cultura de Occidente (Álvarez, Girón, Díaz \& Peña, 1984; Guardo, San Martín, De Gracia \& Palma, 2008; Puente, 2003).

El material de estudio para la adquisición de conocimientos teóricos fueron los diccionarios y manuales médicos de la época (Halilović, 2017). A pesar de que en el período comprendido entre los siglos XI y XVII, el modelo y método que guiaba la formación y la práctica médica en el mundo islámico y europeo procedía de Avicena y su Canon, en el territorio de Al-Ándalus se produjo una excepción. Tomaron mayor importancia otros tratados médicos (Moosavi, 2009), como los de Arib ibn Sa'id (980 d. C.) (Arib Ibn Sa'id, 1983), Abulcasis (1.013 d. C.), Avenzoar (1.077 d. C.) y Averroes (1.126 d.C.) (Al-Majali, 2017; Roldán et al., 2014).

\section{Figuras dedicadas a la asistencia de la salud-enfermedad}

Bajo este marco formativo, cabe establecer una diferenciación según la zona de residencia. En el medio urbano se estima que el desarrollo de la tabiba fue más frecuente. No obstante, también se considera que posiblemente pudo existir una figura denominada mutatabbiba, término derivado de mutatabbib2. En el medio rural sobre todo estuvo presente la figura de la qabila, aunque igualmente se extendió al área urbana para brindar su atención desde los sectores más privilegiados a los menos.

\section{Tabiba}

La tabiba recibió una educación académica desde las aportaciones de la medicina antigua, a través de la tutoría teórica de maestros y maestras con alta cualificación y actualización científica (Aguirre, 1997). A pesar de ser una disciplina muy frecuente entre las mujeres andalusíes, solo se tiene constancia del nombre de seis médicas durante los ocho siglos de historia andalusí, entre las que destacó la ya nombrada Umm al-Hasan, que ejerció la práctica y la docencia de la medicina (Olmo \& Vidal, 2012).

Adquirieron conocimientos anatómicos del cuerpo femenino y fueron expertas en el conocimiento sobre plantas medicinales y sus efectos secundarios. No obstante, gracias a su destreza en la lecto-escritura podían consultar y formarse teóricamente en cualquier campo de la medicina a través de los manuales médicos de la época. Se entrenaban en la práctica cuando acompañaban a la figura formativa en el desempeño de la asistencia sanitaria (Marín, 1993).

Principalmente atendieron a mujeres $y$ menores, y en especial se encargaban de las esclavas del califa o gobernador del momento. Su ámbito de actuación sociosanitario se circunscribió especialmente a usuarios pertenecientes a sectores económicamente privilegiados (Llavero, 2003).

\section{Mutatabbiba}

Se estima que la mutatabbiba pudo acceder al saber por medio de figuras familiares de edad avanzada y como método de aprendizaje primaba la observación y la experiencia. Sus intervenciones podían estar enfocadas al tratamiento de las enfermedades más prevalentes a través de la 
aplicación de sus conocimientos sobre drogas y especias (Aguirre, 1997; Marín, 2006). Se la consideraría médica práctica experimentada por la vía de formación y desempeño de su práctica asistencial, un tanto más popular, lo que en ocasiones podría complicar al lector la diferenciación con las otras figuras pseudoprofesionalizantes, también populares y vinculadas a la elaboración de pócimas y ungüentos (Arvide, 2001).

\section{Qabila}

Aunque las figuras anteriores fueron las más conocidas y tratadas por la literatura médica, escrita en las ciudades (Marín, 1993), también existe constancia de la qabila o matrona andalusí. A pesar de la casi inexistencia de fuentes que aborden la situación de esta figura, se sabe que fue muy solicitada sobre todo en el ámbito rural, y en el urbano atendió tanto a los sectores económicamente privilegiados como los que no (Roldán et al., 2014).

La qabila no poseía una formación académica reglada, a diferencia de las tabibas, sino que aprendía de otras mujeres (Domínguez, Elena \& Fernández, 2017). La adquisición de conocimientos estaba ligada a la práctica de la maestra, de manera que incluso podían llegar a vivir juntas durante el período formativo. Cuando había que atender a una mujer las aspirantes a qabila actuaban como ayudantes de la experta, colocándose a los lados y/o detrás de la parturienta (Arib Ibn Sa'id, 1983) (Figura 3). Mientras, además de ayudar en lo requerido observaban el desempeño de la profesional a la vez que escuchaban las explicaciones que iba dando, para pasar poco a poco de la coparticipación a la asunción absoluta de las labores enseñadas (Ortiz, 1999). De esta manera, la qabila transmitía sus conocimientos de atención al parto oralmente y de generación en generación (García \& Claret-García, 2005) ya que por el hecho de ser mujer se le tenía prohibido escribir tratados o kitab (Marín, 2006; Roldán et al., 2014).

\section{FIGURA 3: Representación islámica del parto en una casa árabe}

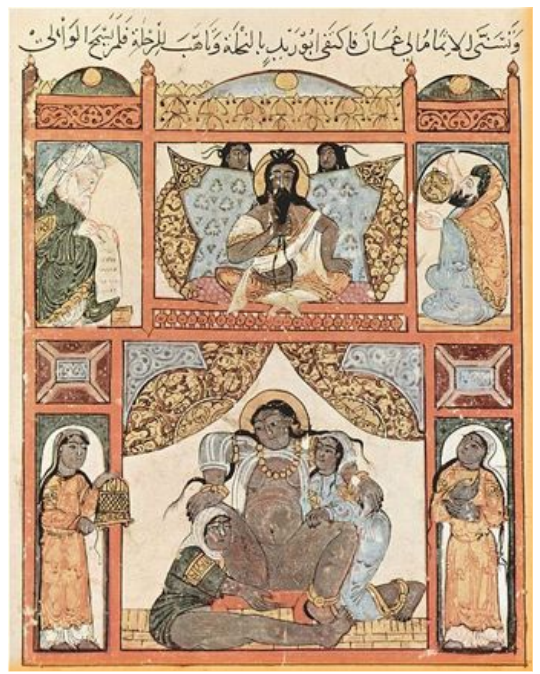

FUENTE: Maqamat de al-Hariri (1054-1122), ilustración realizada por Yahya ibn Mahmud al-Wasiti. Biblioteca Nacional de París. En: Arib Ibn Sa'id (1983) \& http://visualizingbirth.org/medieval-islamic-painting-of-a-queen-giving-birth 


\section{CONCLUSIONES}

La situación formativa de las mujeres de Al-Ándalus fue algo singular para cada una de las culturas que coexistieron en el mismo territorio y durante los siglos VIII-XII. Debido a la asimilación socio-cultural del islam por parte de los pueblos allí presentes, bajo unas normas jurídico-religiosas estrictas sobre el papel pero flexibles en la práctica social, la mujer andalusí pudo formarse y avanzar en los campos del saber.

Aunque la existencia de mujeres dedicadas a la medicina y el cuidado no fue infrecuente, apenas se conocen figuras con nombre $\mathrm{y}$ apellido en los diccionarios biográficos y en las fuentes primarias de la época. Lamentablemente, aunque las mujeres sí pudieron formarse y ejercer su carrera profesional, no pudieron transmitir lo aprendido a través de la escritura, solo de manera oral y en los espacios segregados propios de las mujeres.

Un ejemplo claro del recorrido educativo de mujer dedicada a la medicina fue Umm al-Hasan, quien inicialmente se formó en la lecto-escritura del Corán y después en la poesía y la medicina. Se sabe que una de las profesiones de gran valoración que permitió a las mujeres adquirir otros conocimientos fue la poesía, con lo que la educación de las aspirantes en éste área del saber era esmerada. Es posible que este paso educativo, el de formarse para ser poetisas, fuera frecuente entre las mujeres que posteriormente se instruían en otros campos del saber, línea que podría resultar de gran interés para los próximos estudios.

La figura de la qabila supone a su vez un punto de inflexión, pues el desempeño de su práctica profesional no estuvo determinado por los sectores más o menos privilegiados, pues contrataban sus servicios desde los califas de la corte hasta las personas con menor nivel económico. Además de ser necesaria desde el punto de vista sociosanitario, debido a que el hombre no podía ver por completo el cuerpo de una mujer, la qabila desempeñó una labor brillante, cuestión que le permitió no sólo ejercer funciones asistenciales, sino también de índole jurídica, lo que constituye también un aspecto de gran relevancia a tratar en sucesivos trabajos.

Finalmente, resulta oportuno reflexionar sobre el papel de las mujeres dedicadas a la medicina y el cuidado durante la historia. Es muy probable, que la tradición formativa en Al-Ándalus según ha ido superando las diferentes etapas de la historia, se ha ido adaptando hasta nuestros días, compartiendo grandes similitudes con la educación sanitaria actual.

A pesar de que las figuras femeninas realizaron importantes funciones $y$ aportaciones a la sociedad de la época, realmente no recibieron el reconocimiento personal, familiar, social y cultural que merecían. En algunos casos, sus contribuciones fueron integradas a los tratados médicos escritos por hombres y a la práctica, aunque no bajo su autoría. De esta manera, el presente trabajo adquiere gran relevancia al tratar de recuperar la formación sanitaria de las mujeres en la época andalusí, educación reglada que posteriormente les permitiría dedicarse a la medicina y los cuidados formales en salud, y cuya repercusión se mantiene sobre la situación socio-sanitaria actual.

\section{BIBLIOGRAFÍA}

- Abellán, J. (2006). Poblamiento y sociedad en alÁndalus. En IV-V Jornada de Cultura Islámica 
Almonaster la Real (pp. 13-36). Huelva: Universidad de Huelva.

- Aguilar, V. (1997). Mujeres y repertorios biográficos, biografías y género biográfico en el Occidente Islámico. Estudios Onomástico-Biográficos de $\mathrm{Al}$ Andalus VIII, 127-139.

- Aguirre, L. F. (1997). Sobre el ejercicio de la medicina en al-Andalus: una fetua de Ibn Sahl. Anaquel de Estudios Árabes, 2, 157-160.

- Al-Majali, S. A. M. (2017). Arab Muslims' Medicine and Pharmacy in Andalusia: 138-172 A. D. Research on Humanities and Social Sciences, 7(20), 21-28.

- Álvarez, C., Girón, F., Díaz, A. \& Peña, C. (1984). El niño enfermo en los textos médicos andalusíes. Dynamis, Acta Hispánica ad Medicinae Scientiarumque Historiam Illustrandam, 4, 265-276.

- Arib Ibn Sa'id. (1983). Kitab Khalq al-Janln wa-Tadblr alHabala wa al-Mawludin (DDPP de Córdoba). Córdoba.

- Arvide, M. L. (2001). Un ejemplo de medicina práctica en al-Andalus: el Tratado XIX del Kitab al-taesrif de Abu-l-Qasim al-Zahrawi (c. 936-c. 1013). Dynamis: Acta hispanica ad medicinae scientiarumque historiam illustrandam, 21, 73-92.

- Ávila, M. L. (1989). Las mujeres «sabias» en AlÁndalus. En La mujer en Al-Ándalus: reflejos históricos de su actividad y categorías sociales (pp. 139-184). Madrid, Sevilla: UAM, Eds. Andaluzas Unidas.

- Cabanillas, M. I. (2012). La mujer en Al-Ándalus. En IV Congreso Virtual sobre Historia de las Mujeres (p. 19).

- Puente, C. (2003). Médicos de al-Andalus: perfumes, ungüentos y jarabes: Avenzoar, Averroes, Ibn al-Jatib. Tres Cantos: Nivola.

- Micheli-Serra, A. (2002). Notas sobre la medicina del antiguo Islam. Gac Méd Méx, 138(3), 5.

- Domínguez, A. (2013). España. Tres milenios de historia. Madrid: Marcial Pons Ediciones de Historia.

- Domínguez, P., Elena, A. \& Fernández, S. (2017). Alta Edad Media, siglos V-X: Salud y cuidados realizados en la cultura árabe. En Los Colegios Profesionales de Enfermería, su función social e institucional (Colegio de Enfermería de Salamanca). Salamanca.

- Edriss, H., Rosales, B. N., Nugent, C., Conrad, C. \& Nugent, K. (2017). Islamic Medicine in the Middle Ages. The American Journal of the Medical Sciences, 354(3), 223-229. https://doi.org/10.1016/j.amjms.2017.03.021.

- Fernández, A. \& Mañá, R. (2016). El legado islámico en los cuidados psiquiátricos. El Maristán de Granada. Cultura de los Cuidados Revista de Enfermería y Humanidades,

https://doi.org/10.14198/cuid.2016.45.06.

- García, M. J. \& Claret-García, A. (2005). Las funciones de la matrona en el mundo antiguo y medieval. Una mirada desde la Historia. Matronas Profesión, 6(1), 11-18. - Guardo, A. J., San Martín, G., De Gracia, A. \& Palma,
J. B. (2008). El despertar de la medicina árabe en la Edad Media: algunos de sus médicos. Revista de la Asociación Médica Argentina, 121(2).

- Guichard, P. (1998). Al-Ándalus. Estructura antropológica de una sociedad islámica en occidente. Granada: Universidad de Granada.

- Halilović, S. (2017). Islamic Civilization in Spain - a Magnificient Example of Interaction and Unity of Religion and Science. Psychiatria Danubina, 29(1), 6472.

- Lévi-Provençal, E. (1953). La civilización árabe en España. Buenos Aires: Espasa Calpe.

- Llavero, E. (2003). La Medicina Arabe (Siglos-VII-XI). En Fundación Canaria Orotava de Historia de la Ciencia, Ciencia y cultura en la Edad Media (Vol. Actas VIII y X). Canarias: Consejería de Educación, Cultura y Deportes del Gobierno de Canarias.

- López, G. (1992). Las mujeres andalusíes en el discurso y en la práctica religiosa. En Al-Ándalus: Mujeres, sociedad y religión (pp. 33-62). Málaga: Universidad de Málaga.

- Bernis, M. (1956). La ciencia hispano-árabe. Temas Españoles, $235 . \quad$ Recuperado de http://www.filosofia.org/mon/tem/es0235.htm.

- Marín, M. (1993). Las mujeres en al-Andalus: fuentes e historiografía. En C. del Moral, Arabes, judías y cristianas: mujeres en la Europa medieval (pp. 35-52). Granada: Feminae.

- Marín, M. (2006). Los saberes de las mujeres. En M. Marín, Vidas de mujeres andalusíes (pp. 188-189). Málaga: Sarriá.

- Marín, M. (2010). Derecho islámico medieval y fronteras de género: reflexiones sobre textos de AlBurzuli (m. 841/1438). Clepsydra, 9, 21-40.

- Martín, M. A., Pérez, P. F. \& Sabado, J. T. (2012). Mujer y cuidados: ¿historia de una relación natural? Cultura de los cuidados, 7(13), 36-39. https://doi.org/10.14198/cuid.2003.13.07.

- Mesned, M. S. (2007). El estatus de la mujer en la sociedad árabo-islámica medieval entre oriente y occidente. Editorial de la Universidad de Granada, Granada.

- Moosavi, J. (2009). The Place of Avicenna in the History of Medicine. Avicenna Journal of Medical Biotechnology, 1(1), 3-8.

- Lachiri, N. (1993). La vida cotidiana de las mujeres en Al-Ándalus. En C. del Moral, Árabes, judías y cristianas: mujeres en la Europa medieval. Granada: Universidad de Granada.

- Olmo, A. \& Vidal, F. (2012). Dos modelos de mujeres «sabias» andalusíes en la Loja nazarí. En IV Congreso Virtual sobre Historia de las Mujeres.

- Roldán, M. F. J., Calero, M. Á., Pérez, R. E. M., Calama, A. M. S., Higuera, A. T. \& Concepción, M. B. A. (2014). La «qabila»: historia de la matrona olvidada de alAndalus (siglos VIII-XV). Matronas profesión, (1), 2-8. 


\section{Cultura de los Cuidados}

- Said-Farah, M. (2008). El maristán y Al-Madrasa: Hospital-Escuela de Medicina (II). Archivos de la Sociedad Española de Oftalmología, 83(4), 277-280. https://doi.org/10.4321/S0365-66912008000400012.

- Seila de Castro. (2015). Matrimonios interreligiosos y pensiones (nafaqāt) en el derecho islámico. Su reflejo en el Kitāb al-Nafaqāt del andalusí Ibn Rašīq (s. XI). En VII Congreso virtual sobre Historia de Las Mujeres (pp. 95-112). Universidad de Granada.

- Ortiz, T. (1999). Las matronas y la transmisión de saberes científicos sobre el parto. Arenal, 6(1), 55-79.

- Valdeón, J. (2006). La Reconquista: El concepto de España: Unidad y Diversidad. Pozuelo de Alarcón: Espasa Calpe.

\section{NOTAS}

(1) Al-Mawali: Población magrebí, aunque también hispana en menor medida, que se unió a los Omeyas. Estos últimos proporcionaron a los Al-mawali puestos administrativos como estrategia política para contrarrestar el poder de las estructuras tribales árabes.

(2) Mutatabbib: En la jerarquía de cualificación médica del momento, seguía al médico o tabib, fue considerado médico práctico. Dado que únicamente se ha descrito en la literatura en términos masculinos, sólo es posible teorizar a cerca de una extrapolación de Esta figura a la ocupación femenina, a diferencia de lo que ocurre con el tabib, para el que sí se encuentra recogido el término tabiba en la bibliografía. 